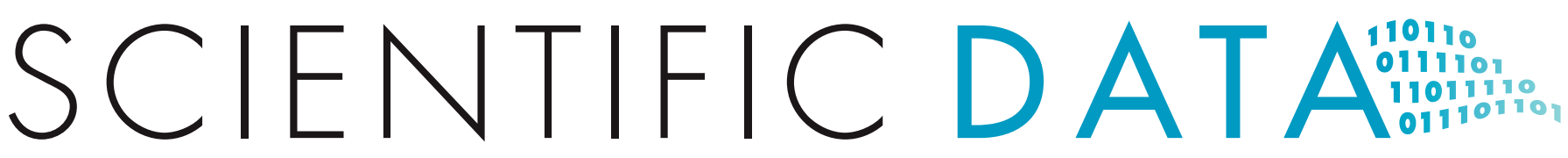

\title{
OPEN Analysis: A systematic review and meta-analysis of seroprevalence surveys of ebolavirus infection
}

Received: 26 May 2016 Accepted: 15 December 2016

Published: 31 January 2017
Hilary Bower ${ }^{1}$ \& Judith R. Glynn ${ }^{1}$

Asymptomatic ebolavirus infection could greatly influence transmission dynamics, but there is little consensus on how frequently it occurs or even if it exists. This paper summarises the available evidence on seroprevalence of Ebola, Sudan and Bundibugyo virus IgG in people without known ebolavirus disease. Through systematic review, we identified 51 studies with seroprevalence results in sera collected from 1961 to 2016 . We tabulated findings by study population, contact, assay, antigen and positivity threshold used, and present seroprevalence point estimates and $95 \%$ confidence intervals. We classified sampled populations in three groups: those with household or known case-contact; those living in outbreak or epidemic areas but without reported case-contact; and those living in areas with no recorded cases of ebolavirus disease. We performed meta-analysis only in the known case-contact group since this is the only group with comparable exposures between studies. Eight contact studies fitted our inclusion criteria, giving an overall estimate of seroprevalence in contacts with no reported symptoms of $3.3 \%(95 \% \mathrm{Cl} 2.4-4.4, P<0.001)$, but with substantial heterogeneity.
${ }^{1}$ Department of Infectious Disease Epidemiology, London School of Hygiene \& Tropical Medicine, London WC1E 7HT, UK. Correspondence and requests for materials should be addressed to H.B. (email: hilary.bower@lshtm.ac.uk). 


\section{Introduction}

Knowing if ebolavirus infection manifests asymptomatically is critical to understanding its spread and to estimating the role herd immunity could have in reducing transmission. Investigating unrecognised infections could also help in the development and targeting of vaccines. However, despite a surprisingly large number of investigations into the seroprevalence of ebolavirus IgG since the first outbreak in Yambuku, Zaire (now Democratic Republic of Congo) ${ }^{1-51}$, consensus on results has proved elusive. The main reasons for this are the range of findings, positive results in unexpected locations, and a lack of confidence in immunofluorescence antibody (IFA) tests used in early studies.

Concerns about IFA specificity stem largely from studies showing positive results in populations expected to be negative, although the most frequently cited-in 200 Panamanian Indians with no known exposure-found only one Ebola virus IgG positive on a high cut-off giving a specificity of $99.5 \%{ }^{4}$. Unexpected seropositivity has also been seen in African countries without reported cases of ebolavirus disease (EVD) such as the Central African Republic, Cameroon and Zimbabwe, only some of which can be attributed to using low test cut-offs. But, as some ELISA-based studies have produced similar findings ${ }^{37,38}$, these positive results may indicate zoonotic exposure with filoviruses or unrecognised human-to-human transmission rather than poor specificity.

'Asymptomatic' status can only be defined for a certain period, such as during an outbreak, though excluding mild symptoms is difficult. In outbreak areas asymptomatic subjects could have experienced unrecognised symptomatic EVD in the past so, even apart from problems with the test, ebolavirus antibody seropositivity does not necessarily mean asymptomatic infection.

We aimed to provide an up-to-date and easily accessible overview of serological findings to date, to help researchers contextualise studies prompted by the 2014-16 West Africa epidemic. The most comprehensive review of ebolavirus serology-Kuhn's Filoviruses: A Compendium of 40 years of Epidemiological, Clinical and Laboratory Studies ${ }^{52}$ - covers work to 2008. In addition to reviewing this key reference, we carried out a systematic review of serosurveys in people without symptoms of EVD up to July 2016.

\section{Results}

\section{Characterisation of seroprevalence surveys of IgG antibodies to ebolavirus}

We identified 51 studies covering 84 sample populations reported to have had no symptoms of EVD during the outbreak period, or to have come from populations with no known outbreaks. In total these studies investigated the presence of ebolavirus IgG in 44,147 subjects using samples collected since 1961.

Thirteen studies reported 16 study populations involving 2,664 participants with household or known case-contact ${ }^{5-7,9,12,36,41,42,45,47,49-51}$. Eleven studies reported 17 study populations covering 5,327 participants living in outbreak areas but without reported case-contact ${ }^{5-7,9,14,33,39,40,42,43,46}$. The remaining studies reported on 51 groups involving 36,156 subjects from general populations, often in settings ecologically similar to ebolavirus outbreak areas but without known cases of $\mathrm{EVD}^{1-3,5,8,10,11,13,15-35,37,38,44-46,48,51}$.

Table 1 (available online only) gives a detailed breakdown of the study populations, test methods and results.

\section{Overall estimates of ebolavirus seroprevalence in asymptomatic individuals}

Only the group with known case-contact had exposures that are comparable across studies and are therefore appropriate to combine by meta-analysis. In this group eight study populations fulfilled the inclusion criteria of testing by ELISA or using a IFA cut-off $\geq 1: 64$ (ref. 5,36,41,42,47,49-51). Pooling these results gave an overall estimate of seroprevalence in asymptomatic people with known case-contact of 3.3\% (95\% CI 2.4-4.4, $P<0.001$ ), but with substantial heterogeneity due to three small studies with higher estimates.

In the other two categories-participants living in outbreak areas but without reported case-contact exposure and general populations in areas without known cases of EVD-exposure was either not well characterised or not well known. Even where EVD cases had not been reported, zoonotic exposure or different forms of disease manifestation could not be ruled out. The highly heterogeneous nature of these study populations makes any single summary estimate inappropriate. In outbreak areas estimates ranged from 0.9 to $17 \%$, and in general populations described as unexposed estimates ranged from 0 to $24 \%$.

\section{Evidence of assay validation}

Few teams reported any validation of the assays used. Some studies repeated analyses with the same technique, usually in a US or European laboratory, but only seven of the 51 studies reported validation work through a different diagnostic platform. Of these, two retested a proportion of IFA positives against ELISA, finding close to $100 \%$ consensus ${ }^{26,30}$. Three tested ELISA against western blot of which two found $100 \%$ specificity ${ }^{38,46,53}$; the third did not report results ${ }^{41}$. Another found 77 and $75 \%$ specificity for ELISA against western Blot and IFA respectively ${ }^{34}$, and a further study confirmed IFA results by western blot but did not report results ${ }^{33}$.

Two studies in Sierra Leone included field testing of ELISA assays in PCR-confirmed positive samples from EVD survivors and community controls with no known exposure to EVD cases from the research area. One, using a novel IgG-capture ELISA ${ }^{54}$, found 95.9\% (95\%CI 89.9-98.9\%) sensitivity and 100\% 
specificity (95\%CI 98.9-100\%) using oral fluid samples from 97 survivors and 339 community controls ${ }^{51}$. The other, using the commercially available ALPHA Diagnostics assay, selected a cut-off that gave $96.7 \%$ sensitivity and $97.7 \%$ specificity in serum samples from 30 survivors and 132 community controls ${ }^{50}$.

\section{Discussion}

We identified 51 studies covering 84 sample populations of 44,147 subjects reported to have had no symptoms of EVD during the outbreak period or to come from populations with no known outbreaks. Most data originated from Western and Middle Africa, and were collected during epidemiological investigations around outbreaks, or in serosurveys in countries without outbreaks but with similar ecology and animal hosts, which aimed to map the geographical extent of the virus. Some studies reported retrospective analysis of samples collected for other reasons prior to the first known outbreak in 1976.

An important finding of our review is the extreme heterogeneity of the studied populations and the lack of clarity in describing their exposure levels. We found that while some studies characterised their sample population clearly by level of contact and presence of symptoms, in many the level of contact/ exposure was less clear, and some did not separate results for symptomatic and asymptomatic subjects. This makes comparison of results difficult, and combining results from the majority of the studies impossible. It may also explain the wide variation of findings which have perplexed investigators over time.

Many studies also employed very different cut-offs to define seropositivity meaning a simple review of results can be misleading. For our analysis, we excluded any study that used a cut off below $\geq 1: 64$ for the studies using IFA, based on the advice in the literature, but there is no definitive evidence that this is an appropriate threshold. The cause of low IFA titre and whether it reflects false positives, or waning antibody response resulting from historical infection which may or may not have been symptomatic, has been frequently discussed. Recently 10 of 12 survivors from Yambuku were reported to have varying degrees of EBOV GP and NP reactivity by ELISA, 40 years after the outbreak ${ }^{55}$. Other studies have shown positive ELISA results in survivors up to 11 years after infection, but neither reported IFA results for comparison $^{56}$

There is no international reference measurement procedure for ebolavirus antibodies and the World Health Organisation has acknowledged the urgent need for one. Interestingly, given the scepticism often expressed regarding the specificity of IFA techniques in ebolavirus serology, a WHO collaborative study undertaken in 2015 to identify an interim reference standard found IFA no less specific or sensitive than the other methods employed, but only a few samples were tested ${ }^{57}$.

There are several limitations to the work presented here. The full information necessary for precision or clear interpretation was often not available. To pursue as high quality research as possible, we have focussed on publications that have undergone peer review and did not search grey literature. With the exception of Kuhn et $a .^{52}$, which has been the standard reference on filovirus seroprevalence surveys to date, we did not search books. In addition to the limitations of the studies themselves noted above and in Table 1, we also note that the distinction of symptomatic and asymptomatic in the papers relied on selfreported health status, which may not be reliable.

To conclude, we present here a comprehensive updated review of seroprevalence surveys for ebolavirus infection in order to better understand the variation in rates found. We highlight the urgent need for validated standardised assays and for detailed characterisation of study population exposures to enable more generalizable estimates of the extent of asymptomatic ebolavirus infection to be made.

\section{Methods}

\section{Search strategy and systematic review}

A systematic search was done in PubMed to identify peer-reviewed papers presenting original data on ebolavirus infection seroprevalence using the following search string:

ebola AND (asymptom* OR antibod* OR IgG OR immun* OR ELISA OR serol ${ }^{\star}$ ) NOT vacc ${ }^{\star}$ NOT immuniz ${ }^{\star}$ AND (Humans[Mesh])

No limitations were placed on language or location of study. Reference lists of the most comprehensive review to date $\mathrm{s}^{52}$ and other papers were also reviewed. Although the focus of interest was data on subjects reported not to have symptoms at the time of an outbreak, we included papers reporting seroprevalence in all populations apart from those with diagnosed EVD in the initial review to ensure relevant studies were not missed.

The search produced 355 citations which were reviewed by title and abstract. Inclusion criteria were: investigation of any African species of ebolavirus immunoglobulin G (ie. not Reston) in individuals without ebolavirus symptoms or in general population groups, with information on denominators and seropositivity and description of those tested. The same search but limited to 2008 to 2016 was rerun on Web of Science; references prior to 2008 were checked against Kuhn et al's list ${ }^{52}$. Four additional citations were found on Web of Science but none were retained for detailed reading. Six citations for papers not already included were identified from reference lists and retained for detailed reading. 


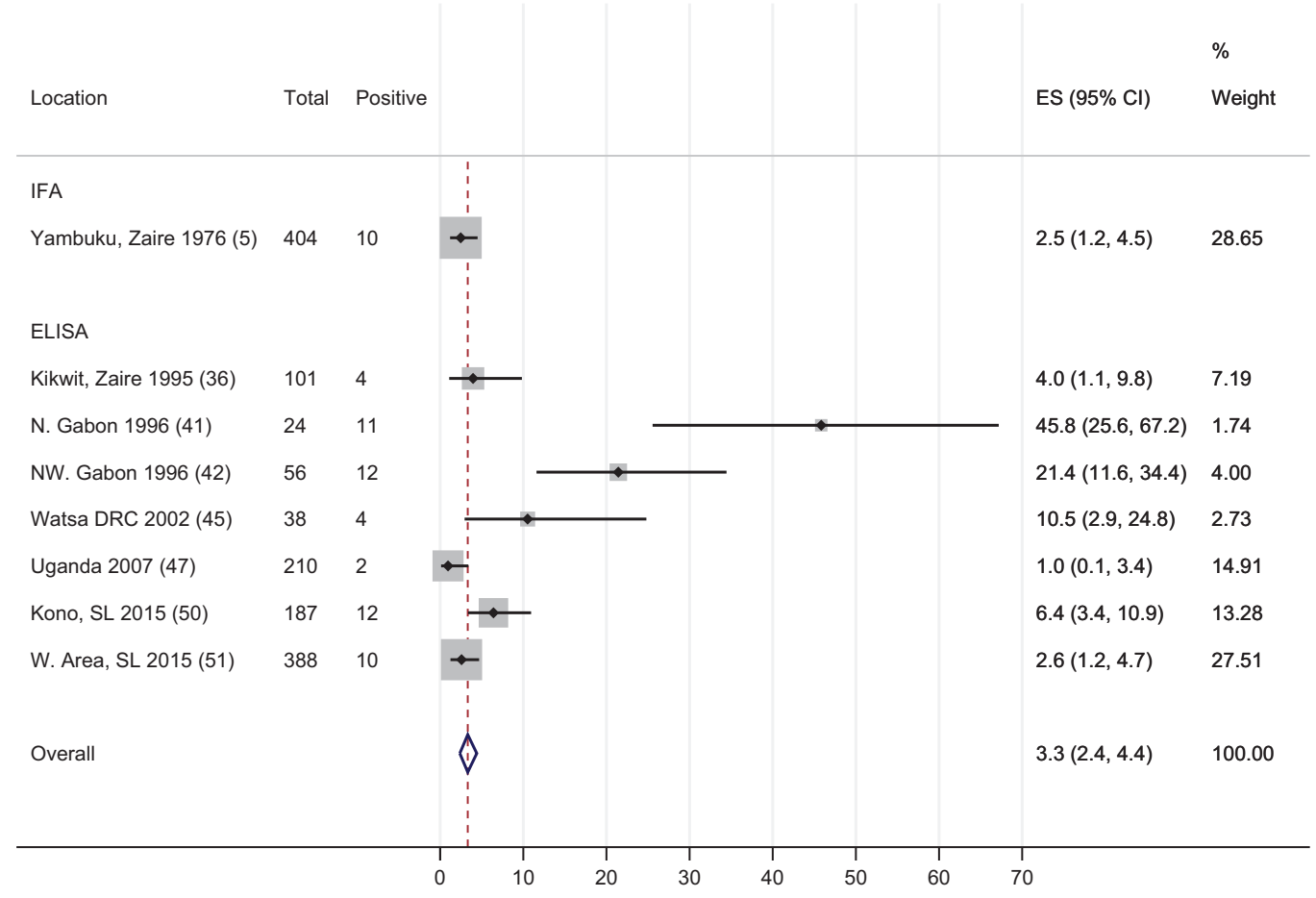

Figure 1. Forest plot and meta-analysis of seroprevalence of ebolavirus IgG among contacts of EVD cases reported to be asymptomatic during the outbreak period. Further details of each included study are given in Table 1. Legend: Ref: reference number; IFA: Immunofluorescence Assay; ELISA: Enzyme-linked immunosorbent assay; ES: Estimated proportion; N, NW: North, Northwestern; SL: Sierra Leone; W. Area: Western Area Province. Note: Zaire now Democratic Republic of Congo; Rhodesia now Zimbabwe.

Total citations: 365 of which 297 (81\%) discarded for the following reasons:

- Detailed immunology or genetics with no relevant data collection for seroprevalence

- Description of acute phase diagnosis and/or investigation of convalescent subjects

- Epidemiology and/or treatment of symptomatic confirmed cases without investigation of non-case populations

- Investigations on sample populations without identifiable non-symptomatic individuals

- Studies examining immune response related to vaccination trials

- Review/comment articles without original data

- Modelling papers without original data

- Preliminary or duplicate reports of the same research study/data.

Sixty-eight papers were read in detail after which a further 20 were discarded for the reasons above. Data extracted from the remaining 48 papers included date of sera collection, composition of study population(s) in terms of exposure, location, selection process and any other defining characteristics, assay type, technique and antigens used, positivity threshold, number of participants per population type, number/proportion of IgG positive individuals, and any information on repeatability or test validity. All selected papers were scrutinised by both authors independently and results discussed and reconciled.

The last search was made on 31 July 2016. Two presentations from the 2016 Conference on Retroviruses and Opportunistic Infections (CROI, Feb. 2016) and one from the 8th International Symposium on Filoviruses (Sept. 2016) describing findings from the 2014-2016 outbreak were also included. A paper reporting one of the CROI presentations has subsequently been published (Nov 2016) and is referenced.

\section{Categorisation of exposure}

Many of the studies reported results on sub-populations with different exposures. To reduce heterogeneity for analysis we categorised these sub-populations under three broad headings according to the extent of exposure: household or known case-contact; living in outbreak areas but without reported case-contact; and subjects drawn from general populations in locations without known EVD. Where study populations were reported to include symptomatic cases and gave enough information to identify these cases, we removed them and recalculated results. 


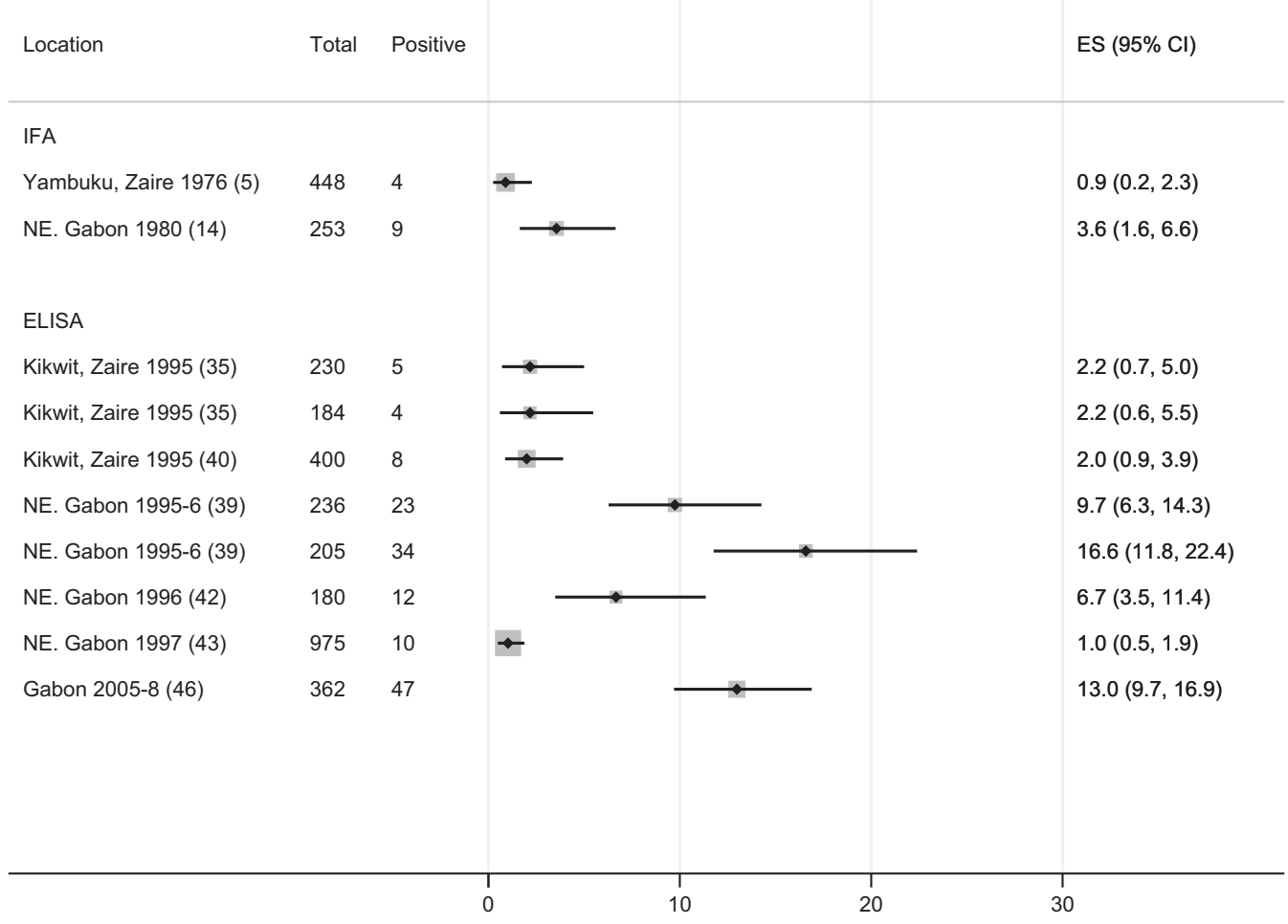

Figure 2. Forest plot of seroprevalence of ebolavirus IgG in individuals reported to be asymptomatic during the outbreak period, recruited in areas with known EVD cases, excluding direct contacts of EVD cases. Further details of each included study are given in Table 1. Legend: Ref: reference number; ES: Estimated proportion; IFA: Immunofluorescence Assay ELISA: Enzyme-linked immunosorbent assay; DRC: Democratic Republic of Congo; N, NE: North, Northeastern.

We excluded one study of PCR negative 'suspects' with close, no or unknown contact exposure due to lack of information on symptom status ${ }^{58}$. In two other studies, sub-groups were not included in the table because they were reported to include symptomatic cases but gave insufficient information to allow recalculation of the seroprevalence estimate excluding those with symptoms ${ }^{1,18}$.

\section{Interpretation of seropositivity}

We have recorded seropositivity results by antigen species where reported; where results were not reported by species, we record positivity to 'ebolaviruses'. 'Overall' positivity is noted where it was reported or where it was possible to rule out double-counting.

To expose the problem of the different positivity thresholds used, we have recorded all studies and their reported cut-off in Table 1. Study characteristics and results have also been formatted as a machinereadable open access dataset (Data Citation 1).

\section{Data visualisation}

To summarise the data visually and present 95\% confidence intervals, we created Forest plots for each of the three exposure categories (Figs 1,2,3) which allow results to be compared in the different contact groups. To address the problem of varying thresholds, we included only those IFA studies that reported results according to the 1:64 titre cut-off cited as more stringent by WHO and others ${ }^{5,18,21,59}$, or which reported enough detail for this threshold to be applied. For ELISA studies, the range of methods used to define positivity was too wide to assign a common threshold so all have been included in the Forest plots, with their method of defining the cut-off detailed in Table 1 (available online only).

\section{Statistical analyses}

We performed a meta-analysis using the Freeman Tukey arcsine square root transformation method and 'fixed effects' (weighted average) inverse variance (metaprop, STATA ${ }^{60}$ ) on the eight study populations with known-case contact. We chose a 'fixed effects' (weighted average) model as contact should give 


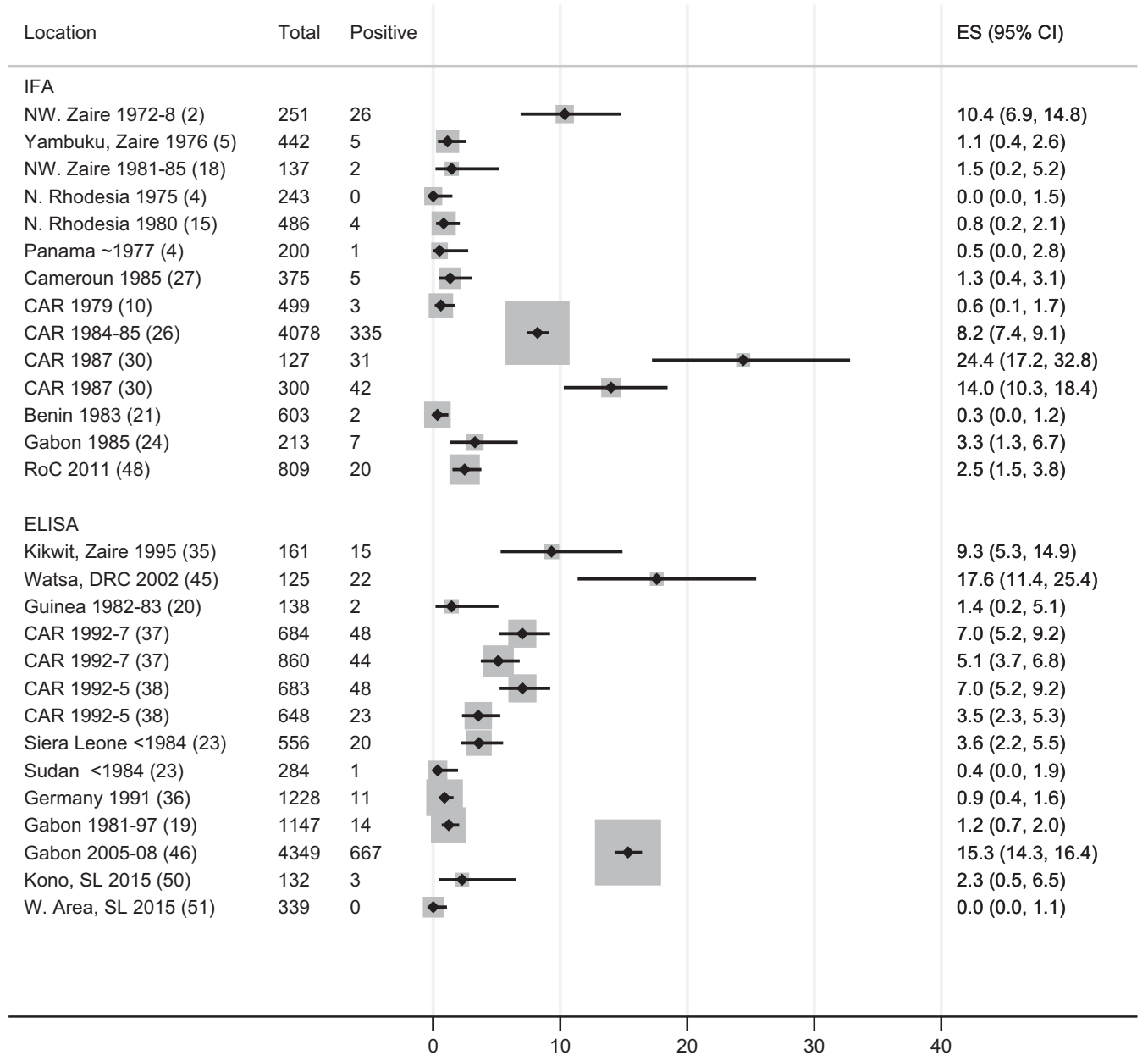

Figure 3. Forest plot of seroprevalence of ebolavirus IgG in general populations living in areas without reported EVD cases. Further details of each included study are given in Table 1. Legend: Ref: reference number; IFA: Immunofluorescence Assay; ELISA: Enzyme-linked immunosorbent assay; ES: Estimated proportion; IFA: Immunofluorescence Assay; ELISA: Enzyme-linked immunosorbent assay; DRC: Democratic Republic of Congo; RoC: Republic of Congo; CAR: Central African Republic; N, NW: North, Northwestern. Note: Zaire now Democratic Republic of Congo; Rhodesia now Zimbabwe.

similar risks in different contexts, and because random effects models give too much weight to small studies $^{61}$. We present an pooled summary estimate for the group with known contact exposure (Fig. 1). We do not show summary estimates for the groups covering subjects living in outbreak areas but without reported case-contact, or drawn from general populations in locations without known EVD (Figs 2 and 3) as these populations are likely to have very different exposure levels so an overall summary estimate of prevalence would be meaningless.

\section{References}

1. Tignor, G. H., Casals, J. \& Shope, R. E. The yellow fever epidemic in Ethiopia, 1961-1962: retrospective serological evidence for concomitant Ebola or Ebola-like virus infection. Transactions of the Royal Society of Tropical Medicine and Hygiene 87, 162 (1993).

2. van der Groen, G. \& Pattyn, S. R. Measurement of Antibodies to Ebola Virus in Human-Sera from Nw-Zaire. Annales de la Societe belge de medecine tropicale 59, 87-92 (1979).

3. Neppert, J., Gohring, S., Schneider, W. \& Wernet, P. No Evidence of Lav Infection in the Republic-of-Liberia, West-Africa, in the Year 1973. Blut 53, 115-117 (1986).

4. van der Groen, G., Johnson, K., Webb, F., Wulff, H, Lange, J. in Ebola Virus Haemorrhagic Fever, edPattyn S. R.)

141-142Elsevier/North-Holland Biomedical Press, (1978).

5. The International Commission. Ebola Haemorrhagic Fever in Zaire. Bulletin WHO (1976).

6. WHO International Study Team. Ebola haemorrhagic fever in Sudan, 1976. Report of a WHO/International Study Team. Bulletin of the World Health Organization 56, 247-270 (1978).

7. Heymann, D. L. et al. Ebola Hemorrhagic Fever: Tandala, Zaire, 1977-1978. Journal of Infectious Diseases 142, 372-376 (1980). 
8. Knobloch, J., Albiez, E. J. \& Schmitz, H. A serological survey on viral haemorrhagic fevers in Liberia. Annales de l'Institut Pasteur/ Virologie 133, 125-128 (1982).

9. Baron, R. C., McCormick, J. B. \& Zubeir, O. A. Ebola virus disease in southern Sudan: hospital dissemination and intrafamilial spread. Bull World Health Organ 61, 997-1003 (1983).

10. Saluzzo, J. F., Gonzalez, J. P., Herve, J. P., Georges, A. J. \& Johnson, K. M. Preliminary note on the presence of antibodies to Ebola virus in the human population in the eastern part of the Central African Republic. Bulletin de la Societe de pathologie exotique et de ses filiales 73, 238-241 (1980).

11. Bouree, P. \& Bergmann, J.-F. Ebola Virus Infection in Man: A Serological and Epidemiological Survey in the Cameroons. The American Journal of Tropical Medicine and Hygiene 32, 1465-1466 (1983).

12. Smith, D. H. et al. Marburg-Virus Disease in Kenya. The Lancet 319, 816-820 (1982).

13. Johnson, B. K. et al. Antibodies against haemorrhagic fever viruses in Kenya populations. Transactions of the Royal Society of Tropical Medicine and Hygiene 77, 731-733 (1983).

14. Ivanoff, B. et al. Haemorrhagic fever in Gabon. I. Incidence of Lassa, Ebola and Marburg viruses in Haut-Ogooue. Transactions of the Royal Society of Tropical Medicine and Hygiene 76, 719-720 (1982).

15. Blackburn, N. K., Searle, L. \& Taylor, P. Viral haemorrhagic fever antibodies in Zimbabwe schoolchildren. Transactions of the Royal Society of Tropical Medicine and Hygiene 76, 803-805 (1982).

16. Talani, P. K., JD., Gromyko, AI., Nanga-Maniane, J., Yala, F. \& Bodzongo, D. Prevelence des anticorps anti-fievres hamorragiques d'origine virale dans la region du Pool (Congo-Brazzaville) Médecine d'Afrique Noire 46 (1999).

17. Van der Waals, F. W., Pomeroy, K. L., Goudsmit, J., Asher, D. M. \& Gajdusek, D. C. Hemorrhagic fever virus infections in an isolated rainforest area of central Liberia. Limitations of the indirect immunofluorescence slide test for antibody screening in Africa. Tropical and geographical medicine 38, 209-214 (1986).

18. Jezek, Z., Szczeniowski, M. Y., Muyembe-Tamfum, J. J., McCormick, J. B. \& Heymann, D. L. Ebola between Outbreaks: Intensified Ebola Hemorrhagic Fever Surveillance in the Democratic Republic of the Congo, 1981-1985. Journal of Infectious Diseases 179, S60-S64 (1999).

19. Lahm, S. A., Kombila, M., Swanepoel, R. \& Barnes, R. F. Morbidity and mortality of wild animals in relation to outbreaks of Ebola haemorrhagic fever in Gabon, 1994-2003. Transactions of the Royal Society of Tropical Medicine and Hygiene 101, 64-78 (2007).

20. Boiro, I. et al. Clinico-epidemiologic and laboratory research on hemorrhagic fevers in Guinea. Bulletin de la Societe de pathologie exotique et de ses filiales 80 607-612 (1987).

21. Gonzalez, J. P. Ebola Virus Circulation in Africa: a balance between clinical expression and epidemiological silence. Epidemiologie 98, 210-217 (2005).

22. Rodhain, F. et al. Arbovirus infections and viral haemorrhagic fevers in Uganda: a serological survey in Karamoja district, 1984. Transactions of the Royal Society of Tropical Medicine and Hygiene 83, 851-854 (1989).

23. Slenczka, W., Rietschel, M., Hoffmann, C. \& W, S. Seroepidemiologische Untersuchungen über das Vorkommen von Antikörpern gegen Marburg und Ebola Virus in Afrika. Mitt. Österr. Ges. Tropical Med. Parasitol 6, 53-60 (1984).

24. Meunier, D. M. Y., Dupont, A., Madelon, M. C., Gonzalez, J. P. \& Ivanoff, B. Surveillance sérologique des fièvres hémorragiques virales dans le Haut-Ogooue (Gabon). Annales de l'Institut Pasteur / Virologie 138, 229-235 (1987).

25. Meunier, D. M. et al. Current serologic data on viral hemorrhagic fevers in the Central African Republic. Bulletin de la Societe de pathologie exotique et de ses filiales 80, 51-61 (1987).

26. Johnson, E. D., Gonzalez, J. P. \& Georges, A. Haemorrhagic fever virus activity in equatorial Africa: distribution and prevalence of filovirus reactive antibody in the Central African Republic. Transactions of the Royal Society of Tropical Medicine and Hygiene 87, 530-535 (1993).

27. Paix, M. A. et al. Serological study of the virus responsible for hemorrhagic fever in an urban population of Cameroon. Bulletin de la Societe de pathologie exotique et de ses filiales 81, 679-682 (1988).

28. Gonzalez, J. P. et al. Antibody prevalence against haemorrhagic fever viruses in randomized representative Central African populations. Research in virology 140, 319-331 (1989).

29. Tessier, S. F., Rollin, P. E. \& Sureau, P. Viral haemorrhagic fever survey in Chobe (Botswana). Transactions of the Royal Society of Tropical Medicine and Hygiene 81, 699-700 (1987).

30. Johnson, E. D., Gonzalez, J. P. \& Georges, A. Filovirus activity among selected ethnic groups inhabiting the tropical forest of equatorial Africa. Transactions of the Royal Society of Tropical Medicine and Hygiene 87, 536-538 (1993).

31. Tomori, O., Fabiyi, A., Sorungbe, A., Smith, A. \& McCormick, J. B. Viral hemorrhagic fever antibodies in Nigerian populations. Am J Trop Med Hyg 38, 407-410 (1988).

32. Mathiot, C. C., Fontenille, D., Georges, A. J. \& Coulanges, P. Antibodies to haemorrhagic fever viruses in Madagascar populations. Transactions of the Royal Society of Tropical Medicine and Hygiene 83, 407-409 (1989).

33. US Centers for Disease Control. Update: filovirus infection associated with contact with nonhuman primates or their tissues. MMWR. Morbidity and mortality weekly report 39, 404-405 (1990).

34. Becker, S., Feldmann, H., Will, C. \& Slenczka, W. Evidence for Occurrence of Filovirus Antibodies in Humans and Imported Monkeys-Do Subclinical Filovirus Infections Occur Worldwide. Medical microbiology and immunology 181, 43-55 (1992).

35. Busico, K. M. et al. Prevalence of IgG Antibodies to Ebola Virus in Individuals during an Ebola Outbreak, Democratic Republic of the Congo, 1995. Journal of Infectious Diseases 179, S102-S107 (1999).

36. Rowe, A. K. et al. Clinical, virologic, and immunologic follow-up of convalescent Ebola hemorrhagic fever patients and their household contacts, Kikwit, Democratic Republic of the Congo. Journal of Infectious Diseases 179, S28-S35 (1999).

37. Nakounne, E., Selekon, B. \& Morvan, J. Microbiological surveillance: viral hemorrhagic fever in Central African Republic: current serological data in man. Bulletin de la Societe de pathologie exotique (1990) 93, 340-347 (2000).

38. Gonzalez, J. P., Nakoune, E., Slenczka, W., Vidal, P. \& Morvan, J. M. Ebola and Marburg virus antibody prevalence in selected populations of the Central African Republic. Microbes and Infection 2, 39-44 (2000).

39. Georges, A. J. et al. Ebola hemorrhagic fever outbreaks in Gabon, 1994-1997: epidemiologic and health control issues. The Journal of infectious diseases 179(Suppl 1): S65-S75 (1999).

40. Tomori, O. et al. Serologic Survey among Hospital and Health Center Workers during the Ebola Hemorrhagic Fever Outbreak in Kikwit, Democratic Republic of the Congo, 1995. Journal of Infectious Diseases 179, S98-S101 (1999).

41. Leroy, E. M. et al. Human asymptomatic Ebola infection and strong inflammatory response. The Lancet 355, 2210-2215 (2000).

42. Bertherat, E., Renaut, A., Nabias, R., Dubreuil, G. \& Georges-Courbot, M. C. Leptospirosis and Ebola virus infection in five gold-panning villages in northeastern Gabon. The American Journal of Tropical Medicine and Hygiene 60, 610-615 (1999).

43. Heffernan, R. T. et al. Low seroprevalence of IgG antibodies to Ebola virus in an epidemic zone: Ogooue-Ivindo region, Northeastern Gabon, 1997. The Journal of infectious diseases 191, 964-968 (2005). 
44. Vladyko, A. S. et al. False-positive reactions in laboratory diagnosis of Lassa, Marburg, and Ebola viral hemorrhagic fevers and AIDS. Russian Progress in Virology 2, 25-30 (1997).

45. Mulangu, S. et al. High prevalence of IgG antibodies to Ebola virus in the Efe pygmy population in the Watsa region, Democratic Republic of the Congo. Bmc Infectious Diseases 16, 263 (2016).

46. Nkoghe, D. et al. Risk factors for Zaire ebolavirus--specific IgG in rural Gabonese populations. The Journal of infectious diseases 204(Suppl 3): S768-S775 (2011).

47. Clark, D. V. et al. Long-term sequelae after Ebola virus disease in Bundibugyo, Uganda: a retrospective cohort study. The Lancet Infectious Diseases 15, 905-912 (2015).

48. Moyen, N. et al. Risk Factors Associated with Ebola and Marburg Viruses Seroprevalence in Blood Donors in the Republic of Congo. PLoS neglected tropical diseases 9, e0003833 (2015).

49. Fallah, M. \& PREVAIL III Research Team. A cohort study of survivors of Ebola Virus Infection in Liberia. Conference on Retroviruses and Opportunistic Infections (CROI). At: http://www.croiwebcasts.org/console/player/29569?mediaType=slide Video\& (2016).

50. Richardson, E. T. et al. Minimally Symptomatic Infection in an Ebola 'Hotspot': A Cross-Sectional Serosurvey. PLoS neglected tropical diseases 10, e0005087 (2016).

51. Glynn, J. B. et al. Asymptomatic infection and unrecognised Ebola Virus Disease, Sierra Leone (Filovirus 2016 Symposium, Antwerp, Belgium (2016).

52. Kuhn, J. H. \& Calisher, C. H. Filoviruses: a compendium of 40 years of epidemiological, clinical and laboratory studies. Springer Science and Business Media, (2008).

53. Becquart, P. et al. High Prevalence of Both Humoral and Cellular Immunity to Zaire ebolavirus among Rural Populations in Gabon. Plos One 5, e9126 (2010).

54. Lambe, T. et al. Detection of Vaccine-Induced Antibodies to Ebola Virus in Oral Fluid. Open forum infectious diseases 3, ofw031 (2016).

55. Remoin, A. et al. Persistent Immune Response in Ebola Survivors from Yambuku Outbreak 40 years after Infection (Filovirus 2016 Symposium, Antwerp, Belgium (2016).

56. Wauquier, N., Becquart, P., Gasquet, C. \& Leroy, E. M. Immunoglobulin G in Ebola outbreak survivors, Gabon. Emerg Infect Dis 15, 1136-1137 (2009).

57. Expert committee on Biological Standardisation. WHO collaborative study to assess the suitability of an interim standard for antibodies to Ebola virus. Report No. WHO/BS/2015.2280 post-ECBS, (World Health Organisation (2015).

58. de La Vega, M. A. et al. Ebola viral load at diagnosis associates with patient outcome and outbreak evolution. The Journal of clinical investigation 125, 4421-4428 (2015).

59. Pattyn, S. R.. Ebola Virus Haemorrhagic Fever: Proceedings of an International Colloquium on Ebola Virus Infection and Other Haemorrhagic Fevers held in Antwerp, Belgium, 6-8 December, 1977. Elsevier/North-Holland Biomedical Press, (1978).

60. Nyaga, V. N., Arbyn, M. \& Aerts, M. Metaprop: a Stata command to perform meta-analysis of binomial data. Archives of Public Health 72, 39 (2014).

61. Higgins J. \& Green S.(eds)in The Cochrane Handbook for Systematic Reviews of Interventions (Version 5.1.0) (The Cochrane Collaboration).

62. Wulff, H. \& Lange, J. Indirect Immunoflouresce for the diagnosis of Lassa Fever Infection. Bulletin of the World Health Organization 52, 429-436 (1975).

63. Johnson, K. M., Elliott, L. H. \& Heymann, D. L. Preparation of polyvalent viral immunofluorescent intracellular antigens and use in human serosurveys. Journal of clinical microbiology 14, 527-529 (1981).

64. Gardner, P. S. \& McQuillin, J. Rapid virus diagnosis, application of immunoflourescence. (Butterworth \& Co, 1974).

65. Bashkirtsev, V. N., Tkachenko, E. A., Dzagurova, T. K. \& Ryl'tseva, E. V. Isolation of strains of the virus of hemorrhagic fever with renal syndrome in cell culture. Voprosy virusologii 29, 497-502 (1984).

66. Emmerich, P. et al. Reverse ELISA for IgG and IgM antibodies to detect Lassa virus infections in Africa. Journal of clinical virology : the official publication of the Pan American Society for Clinical Virology 37, 277-281 (2006).

67. Ksiazek, T. G., West, C. P., Rollin, P. E., Jahrling, P. B. \& Peters, C. J. ELISA for the Detection of Antibodies to Ebola Viruses. Journal of Infectious Diseases 179, S192-S198 (1999).

68. Schoepp, R. J., Rossi, C. A., Khan, S. H., Goba, A. \& Fair, J. N. Undiagnosed acute viral febrile illnesses, Sierra Leone. Emerg Infect Dis 20, 1176-1182 (2014).

69. Boisen, M. L. et al. Multiple circulating infections can mimic the early stages of viral hemorrhagic fevers and possible human exposure to filoviruses in Sierra Leone prior to the 2014 outbreak. Viral immunology 28, 19-31 (2015).

70. Niklasson, B., Peters, C. J., Grandien, M. \& Wood, O. Detection of human immunoglobulins G and M antibodies to Rift Valley fever virus by enzyme-linked immunosorbent assay. Journal of clinical microbiology 19, 225-229 (1984).

71. Rezapkin, G. V., Tkachenko, E. A., Ivanov, A. P., Bashkirtsev, V. N. \& Dzagurova, T. K. Determination of arenavirus antigens and antibodies by solid-phase radioimmunological analysis. Voprosy virusologii 459-462 (1981).

72. Johnson, B. K. et al. Viral haemorrhagic fever surveillance in Kenya, 1980-1981. Tropical and geographical medicine 35, 43-47 (1983).

73. US Centers for Disease Control. Update: evidence of filovirus infection in an animal caretaker in a research/service facility. MMWR. Morbidity and mortality weekly report 39, 296-297 (1990).

74. US Centers for Disease Control. Update: filovirus infections among persons with occupational exposure to nonhuman primates. MMWR. Morbidity and mortality weekly report 39, 266-267, 273 (1990).

75. Alpha Diagnostic International, I. Zaire-Ebola virus nucleoprotein (EBOV-NP) IgG (AE-320520-1) kit and IgM (AE-320530-1) ELISA kit product descriptions. http://www.4adi.com/ (2016).

\section{Data Citations}

1. Bower, H. \& Glynn, J. R. Dryad Digital Repository http://dx.doi.org/10.5061/dryad.gn95r (2016).

\section{Acknowledgements}

We acknowledge with thanks the support of the Wellcome Trust's Enhancing Research Activity in Epidemic Situations (ERAES) programme (Grant No. ER1502).

\section{Author Contributions}

H.B. conceived the review, created the search strategy, retrieved and screened the papers, extracted and compiled the data, created the graphics, carried out the analysis and drafted the paper. J.R.G. reviewed the strategy and analysis, checked all data extraction, and contributed to subsequent drafts of the paper. 


\section{Additional Information}

Table 1 is only available in the online version of this paper.

Competing financial interests: H.B. and J.R.G. declare they have no competing financial interests nor conflicts of interest. Both authors have had had full access to all the data in the study and take responsibility for the integrity of the data and the accuracy of the data analysis.

How to cite this article: Bower, H. \& Glynn, J. R. A systematic review and meta-analysis of seroprevalence surveys of ebolavirus infection. Sci. Data 4:160133 doi: 10.1038/sdata.2016.133 (2017).

Publisher's note: Springer Nature remains neutral with regard to jurisdictional claims in published maps and institutional affiliations.

(c) (i) This work is licensed under a Creative Commons Attribution 4.0 International License. The images or other third party material in this article are included in the article's Creative Commons license, unless indicated otherwise in the credit line; if the material is not included under the Creative Commons license, users will need to obtain permission from the license holder to reproduce the material. To view a copy of this license, visit http://creativecommons.org/licenses/by/4.0

(c) The Author(s) 2017 